\title{
Two positrons can form a chemical bond in $(\mathrm{PsH})_{2}$
}

\author{
Dario Bressaninia \\ Dipartimento di Scienza e Alta Tecnologia, Università dell'Insubria, Via Valleggio 9, I-22100 Como, Italy \\ (Received ---) \\ adario.bressanini@uninsubria.it
}

\begin{abstract}
We show that two positrons can form a chemical bond between two otherwise repelling ions, similarly to what happens to two hydrogen atoms forming a hydrogen molecule. Two positronium hydride atoms (PsH) can form the stable species $(\mathrm{PsH})_{2}$ when the two coupled positrons have opposite spin, while they form an antibonding state if they have the same spin. This is completely analogous to the landmark description by Heitler and London of the formation of a chemical bond in the hydrogen molecule coupling two electrons with opposite spin. This is the first time two positrons are shown to behave like two electrons in ordinary matter, enlarging the definition of what is a chemical bond dating back to Lewis. We suggest a few experimental routes to form and detect such a peculiar molecule.
\end{abstract}

\section{INTRODUCTION}

Positrons, the antimatter version of electrons, are now widely used in many fields, from positron emission tomography (PET) in medical imaging ${ }^{1}$ to structural studies of materials using annihilation spectroscopy ${ }^{2}$ to astrophysics $^{3}$. In the presence of ordinary matter, these antimatter particles can sometimes bind to regular atoms and molecules ${ }^{4}$ forming chemically stable species, before undergoing annihilation, whose radiation is detected, studied, and interpreted. However, the underlying binding mechanism (or mechanisms) of positrons to ordinary matter is far from being theoretically well understood.

Two hydrogen atoms can share their electrons to form a bond producing a hydrogen molecule. In his landmark paper "The atom and the molecule "Lewis" laid the foundation of modern chemical bonding theory and used his model to qualitatively describe the formation of the hydrogen molecule as well as many other molecules. Eleven years later, just after the introduction of modern quantum mechanics, Heitler and London $^{6}$ (HL) provided their groundbreaking quantum mechanical description of the two-electron covalent bond between two hydrogen atoms. Quoting a recent perspective article "Heitler and London had solved the longstanding puzzle of how there could be a strong attraction between neutral atoms, showing that chemical bonding was due to quasi-classical Coulomb interactions and quantum mechanical interference of the atomic electronic wavefunction." Heitler and London, using a primitive version of what would be later called Valence Bond Theory, showed that the wave functions of the two fragments -the two hydrogen atoms- could be combined in two different ways: bonding and antibonding, where the total wave function is either symmetric or antisymmetric with respect to the exchange of the two electrons.

In 1932 Carl Anderson discovered the positron ${ }^{8,9}$, a particle identical to the electron but with a positive charge, whose existence was predicted by Paul Dirac ${ }^{10}$ the year before. Antihydrogen $\overline{\mathrm{H}}$, composed by an antiproton and a positron, has been produced and detected $^{11}$ in 1995 and it is actively studied ${ }^{12}$, and while the antihydrogen molecule $\overline{\mathrm{H}}_{2}$ has not been produced yet, it is believed that the two positrons would behave similarly to the electrons in ordinary $\mathrm{H}_{2}$ forming a chemical bond between the two antihydrogen atoms.

A positron can also bind to ordinary matter to form electronically stable systems (i.e., with a total energy lower than the fragments), before undergoing an annihilation process. The simplest system it can form is Ps, positronium, composed by one positron and one electron. A positron cannot form a stable system with a hydrogen atom but can attach to $\mathrm{H}^{-}$to form positronium hydride (PsH), whose theoretical prediction dates back to Ore's pioneering calculation ${ }^{13}$ in 1951. PsH was finally produced and experimentally detected by Schrader ${ }^{14}$ in 1992. Despite the molecular notation, PsH can be considered a neutral atom with one positron and two electrons orbiting around the nucleus ${ }^{15}$. In fact it is the simplest neutral system with a positron orbiting around a heavy nucleus, and in a very loose sense it can be considered analogous to antihydrogen: the proton and the two electrons of $\mathrm{H}^{-}$collectively play the role of the antiproton, with its negative charge able to bind the positron in $\overline{\mathrm{H}}$. Pushing the analogy to the limit, we can ask if two PsH atoms can interact to form a bound system as two antihydrogen atoms would interact to form $\overline{\mathrm{H}}_{2}$.

In this paper we show that indeed two $\mathrm{PsH}$ atoms can interact to form a locally stable $(\mathrm{PsH})_{2}$ molecule. Similarly to what Heitler and London showed in their 
famous paper, two PsH can interact forming a bonding and an antibonding wave function, depending on the spin state -respectively singlet and triplet- of the two positrons. As the pair of electrons in $\mathrm{H}_{2}$ form a chemical bond between the two otherwise repelling $\mathrm{H}^{+}$, the pair of positrons in $(\mathrm{PsH})_{2}$ form a locally stable molecule with a chemical bond between the two otherwise repelling $\mathrm{H}^{-}$.

In the last few decades a handful of atomic systems containing two positrons have been theoretically predicted $^{16-20}$, although none of them has been experimentally produced yet, but in all cases the two positrons are not the ultimate cause of the binding. $(\mathrm{PsH})_{2}$ is the first system predicted to have a twopositron chemical bond.

\section{METHOD}

Quantum Monte Carlo (QMC) methods can treat the instantaneous correlation between the six leptonic particles exactly and on equal footings, so they represent the ideal technique to study systems containing positrons. Within the Born-Oppenheimer (BO) approximation, we employed the explicitly correlated functional form

$\Psi=\hat{A}(1+\hat{\imath}) \prod_{i=1}^{6} f_{i A}\left(r_{i A}\right) f_{i B}\left(r_{i B}\right) \prod_{i<j} g_{i j}\left(r_{i j}\right)$

as the main building block to describe the interaction between the two $\mathrm{PsH}$ atoms from $\mathrm{R}=12$ bohr to $\mathrm{R}=3.2$ bohr in steps of 0.4 bohr. $\hat{A}$ is the antisymmetrizer operator, $\hat{\imath}$ is the inversion operator, $r_{i X}$ is the distance between particle $i$ and nucleus $\mathrm{X}$, while $r_{i j}$ is the distance between particles $i$ and $j$. We assumed that the electrons are in a singlet state and the total angular momentum is 0 . The positrons can be in a singlet or a triplet state, and the antisymmetrizer operator takes care of the correct spatial symmetry of the wave function. The functional form in equation (1) is the simplest most general function where all two-body terms have been included. The $f_{i X}$ and the $g_{i j}$ functions have the common generic 3parameter exponential Padè form:

$$
f_{i X}(r)=g_{i j}(r)=e^{\frac{a r+b r^{2}}{1+c r}}
$$

The parameters in each function are independent from each other and can assume different values. To reduce the number of nonlinear variational parameters and to help their optimization, we fixed $a$ to the cusp theoretical values of +1 and -1 respectively for the positron-nucleus and electron-nucleus interaction. For the electron-electron and the positron-positron interactions we set $a=1 / 2$ or $a=1 / 4$ for unlike and like spin respectively while for electron-positron interactions we set the cusps to $a=-1 / 2$. We optimized all other parameters. This functional form in previous studies $^{15,21-23}$, was able to describe with great accuracy the essential physics of small electronic and positronic systems.

We used variational Monte Carlo (VMC) to estimate the variational energy. We first roughly optimized the parameters minimizing the mean absolute deviation of the local energy ${ }^{24}$, and then fine-tuned them using an energy optimization procedure. We employed the resulting wave functions in Fixed-Node Diffusion Monte Carlo (DMC) simulations to obtain an upper bound to the exact energy. We estimated the DMC energies using 1000 walkers using a time step of 0.01 hartree $^{-1}$. We have eliminated the time step bias in the minimum of the DMC potential curve using the standard extrapolation to zero. The density distributions have been computed using the second order estimator.

\section{HL TREATMENT OF (PSH) 2}

Following the original HL treatment of the hydrogen molecule, we computed the interaction energy between two PsH atoms as a function of the internuclear distance $\mathrm{R}$, keeping $\Psi(\mathrm{PsH})$ fixed and combining the fragment wave functions to obtain either a spatially symmetric ${ }^{1} \Sigma_{\mathrm{g}}$ state, where the two positrons are in a singlet state with total spin $S=0$, or an antisymmetric ${ }^{3} \Sigma_{\mathrm{g}}$ state, where the two positrons are in a triplet state with total spin $S=1$. Using this rough quantum mechanical treatment, the potential energy curve (PEC) for the singlet state shows a local minimum, and a binding energy (BE) of 0.0011(1) hartree, while the triplet state PEC is completely repulsive. This is analogous to what Heitler and London observed for the $\mathrm{H}_{2}$ molecule. Their calculation was immediately refined by $\mathrm{Wang}^{25}$ who allowed the wave functions of the two fragments to relax, reoptimizing the total wave function. In our case we completely reoptimized the total wave function allowing the four electrons and two positrons of the two $\mathrm{PsH}$ to interact with each other and with the two nuclei.

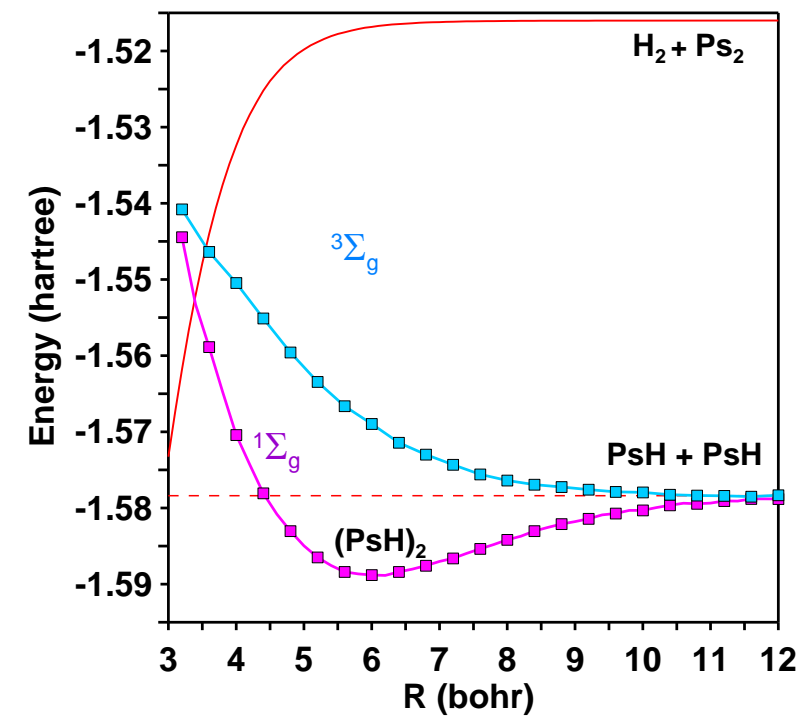


FIG. 1. DMC potential energy curves of two interacting PsH atoms. The singlet state (purple) has a local minimum while the triplet state (cyan) is purely repulsive. The error bars of the individual computed points are smaller than the size of the symbol. The PsH + PsH energy threshold is shown in dashed red while the energy of the $\mathrm{H}_{2}+\mathrm{Ps}_{2}$ dissociation channel is shown in solid red.

Figure 1 and Table I show the diffusion Monte Carlo (DMC) potential energy curve for both the singlet and the triplet positronic states. The attractive (singlet) curve at about $\mathrm{R}=6$ bohr has a minimum 0.0104(1) hartree deep with respect to the $\mathrm{PsH}+\mathrm{PsH}$ dissociation channel, indicating the formation of a chemically stable molecule between two PsH atoms where the two positrons are in a singlet state. On the other hand, the interaction between two PsH atoms where the positrons are in a triplet state is purely repulsive. This is completely analogous to what happens with two hydrogen atoms and likely with two antihydrogen atoms- forming a ${ }^{1} \Sigma_{\mathrm{g}}$ state and a ${ }^{3} \Sigma_{\mathrm{g}}$ state of the hydrogen (or antihydrogen) molecule.

When the two nuclei are at infinite distance, the energy of the fragments ( $\mathrm{PsH}+\mathrm{PsH})$ is $\mathrm{E}=-1.578392$ hartree. When the two systems approach each other, they feel an attractive potential and the potential energy surface shows a local minimum at about $\mathrm{R}=6 \mathrm{bohr}$. To estimate more accurately the position of the equilibrium structure, calculation of the potential energy curve on a finer mesh must be computed. Our DMC calculations in the minimum of the PEC predicts a total energy of 1.5888(1) hartree and a BE of 10.4(1) mhartree.

Table I. DMC energies of the singlet and triplet positronic states of $(\mathrm{PsH})_{2}$ as a function of the internuclear distance. In parenthesis the statistical error on the last digit. Energies are in hartree, distances in bohr.

\begin{tabular}{lcc}
\hline \hline $\mathrm{R}$ & ${ }^{1} \Sigma_{\mathrm{g}}$ DMC energy & ${ }^{3} \Sigma_{\mathrm{g}}$ DMC energy \\
\hline 3.2 & $-1.5444(3)$ & $-1.5408(3)$ \\
3.6 & $-1.5588(3)$ & $-1.5464(2)$ \\
4 & $-1.5704(2)$ & $-1.5505(2)$ \\
4.4 & $-1.5781(2)$ & $-1.5551(2)$ \\
4.8 & $-1.5830(2)$ & $-1.5596(2)$ \\
5.2 & $-1.5864(1)$ & $-1.5634(2)$ \\
5.6 & $-1.5884(1)$ & $-1.5666(2)$ \\
6 & $-1.5888(1)$ & $-1.5687(1)$ \\
6.4 & $-1.5884(1)$ & $-1.5714(2)$ \\
6.8 & $-1.5876(1)$ & $-1.5723(1)$ \\
7.2 & $-1.5866(1)$ & $-1.5743(1)$ \\
7.6 & $-1.5854(1)$ & $-1.5756(1)$ \\
8 & $-1.5842(1)$ & $-1.5764(1)$ \\
8.4 & $-1.5830(1)$ & $-1.5769(1)$ \\
8.8 & $-1.5821(1)$ & $-1.5772(1)$ \\
9.2 & $-1.5814(1)$ & $-1.5776(1)$ \\
9.6 & $-1.5807(1)$ & $-1.5779(1)$ \\
10 & $-1.5803(1)$ & $-1.5779(1)$
\end{tabular}

\begin{tabular}{lll}
10.4 & $-1.5796(1)$ & $-1.5783(1)$ \\
10.8 & $-1.5794(1)$ & $-1.5784(1)$ \\
11.2 & $-1.5791(1)$ & $-1.5784(1)$ \\
11.6 & $-1.5788(1)$ & $-1.5785(1)$ \\
12 & $-1.5788(1)$ & $-1.5783(1)$ \\
\hline \hline
\end{tabular}

The binding energy, although smaller than the $\mathrm{H}_{2}$ binding energy, it is large enough to support a few vibrational states, whose exact number and frequencies could be determined with calculations which are outside the scope of the present work. For $\mathrm{R}<6$ bohr the ${ }^{1} \Sigma_{\mathrm{g}}$ potential curve in Figure 1 becomes repulsive. At approximately $\mathrm{R}=3.6 \mathrm{bohr}$ the potential energy curve intersects the $\mathrm{H}_{2}+\mathrm{Ps}_{2}$ curve, where the system rearranges into a dipositronium molecule $\mathrm{Ps}_{2}$ at infinity and the remaining two electrons forming a hydrogen molecule with the two nuclei.

These calculations prove that $(\mathrm{PsH})_{2}$ is electronically stable with respect to the $\mathrm{PsH}+\mathrm{PsH}$ dissociation channel. For $\mathrm{R}<3.4$ bohr the system dissociates into $\mathrm{H}_{2}$ $+\mathrm{Ps}_{2}$, and while the equilibrium geometry is around 6 bohr-a much larger value-it is possible that the system, even in the vibrational ground state, dissociates by tunneling into $\mathrm{H}_{2}+\mathrm{Ps}_{2}$. The calculation of the tunneling rate and other dynamical properties of this system are beyond the scope of the present study.

\section{THE TWO-POSITRON CHEMICAL BOND}

We now describe the changes in the positronic distribution that allows the formation of a chemical bond. Figure 2 shows the electronic and positronic densities projected on the $z$ axes for two noninteracting and two interacting $\mathrm{PsH}$ atoms positioned 6 bohr apart.

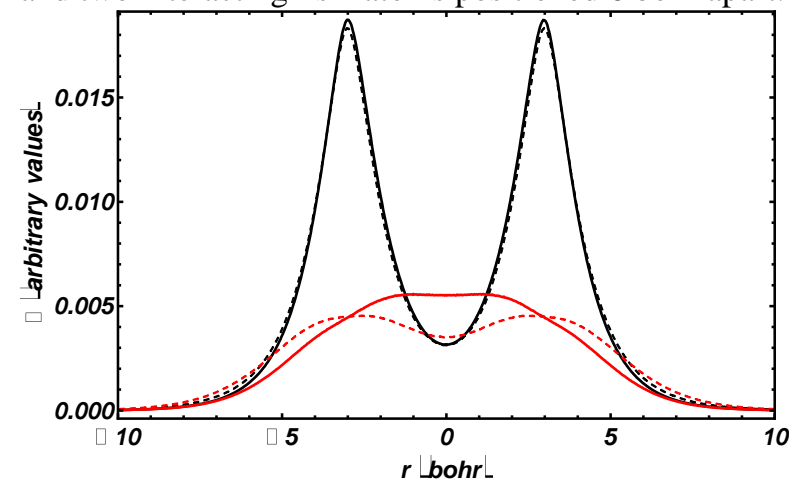

FIG. 2. Electronic (black) and positronic (red) densities projected on the $z$ axes of two noninteracting (dashed) and interacting (solid) $\mathrm{PsH}$ atoms 6 bohr apart. The electronic and positronic densities are normalized respectively to 4 and 2 .

The dashed curves are just the superposition of the electronic and positronic distributions of the two isolated PsH 6 bohrs apart. As soon as the interaction is turned on, the $z$-projected positronic density moves towards the internuclear region producing an accumulation of the positronic density. The electronic density when the interaction is turned on and off is 
practically unchanged, ruling out the possibly that the electrons are responsible for the formation of the chemical bond. On the other hand, the positronic distribution, shown in Figure 2, changes dramatically from the noninteracting to the interacting case. In the noninteracting case each positron moves around its nucleus while, as soon as the interaction is turned on, there is an accumulation of positronic density in the internuclear region, as one would expect from the formation of a chemical bond from the two otherwise repelling $\mathrm{H}^{-}$ions.

A more vivid view of the chemical bond can be seen projecting the electronic and positronic densities of $(\mathrm{PsH})_{2}$ at the equilibrium distance onto the $x z$ plane. The two (chopped) peaks of the electronic distribution are clearly visible in Figure 3.

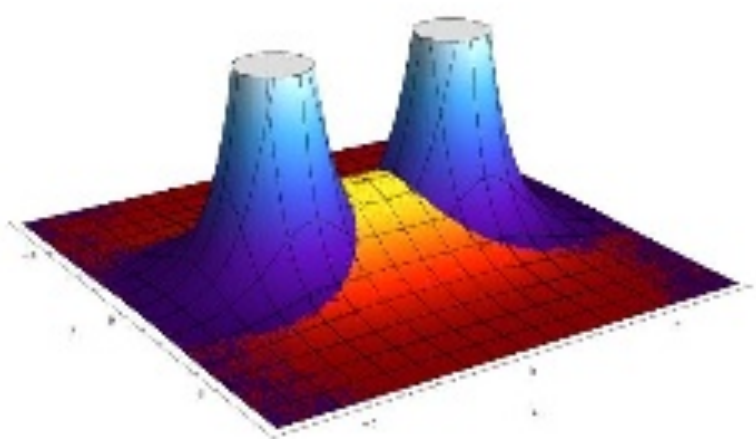

FIG. 3. Electronic (blu tones) and positronic (yellow/red tones) densities of $(\mathrm{PsH})_{2}$ in arbitrary units projected on the $x z$ plane. The electronic density is chopped to show the positronic density on the same graph.

The positron distribution surrounding the two $\mathrm{H}^{-}$ions acts as a positronic glue ${ }^{26,27}$ keeping the system together.

\section{CONCLUSIONS}

We showed that two positrons, coupled in a singled state, can form a chemical bond between two positronium hydride atoms, completely akin to what happens with two electrons in two hydrogen atoms. Ruedenberg ${ }^{28}$ showed that the driving force of the formation of the chemical bond in $\mathrm{H}_{2}$ and $\mathrm{H}_{2}^{+}$is the lowering of the kinetic energy density of the shared electrons in the bonding region. It remains to be seen if the binding mechanism in $(\mathrm{PsH})_{2}$ is the same as in the $\mathrm{H}_{2}$ covalent bond or it is a completely different and new type of bond.

It is interesting to speculate how one could proceed to experimentally produce such a system. The intersection between the PEC of $(\mathrm{PsH})_{2}$ and the $\mathrm{H}_{2}+\mathrm{Ps}_{2}$ curve is above the energy of the fragments $\mathrm{PsH}+\mathrm{PsH}$. This is important to establish the relative stability with respect to the fragments at infinity. In principle it could be possible to form $(\mathrm{PsH})_{2}$ by a collision between two positronium hydride with some mechanism to dissipate the excess energy the collision. However, although PsH has been experimentally produced ${ }^{14}$, unfortunately at present there is no a PsH beam facility available, while Ps and even $\mathrm{Ps}^{-}$beams are now available.

$\mathrm{Ps}_{2}$ molecules have been recently produced and detected implanting intense positron pulses into porous silica film ${ }^{29}$ or on a clean $\mathrm{Al}(111)$ surface $^{30}$ and in principle they could be used to produce $(\mathrm{PsH})_{2}$. We note that the minimum of the PEC corresponds to an internuclear distance $\mathrm{R}$ where the potential curve of the hydrogen molecule is still flat, and the two hydrogen atoms interact only weakly. This means that it is unlikely that an experimental realization of the $(\mathrm{PsH})_{2}$ system could be done by a collision of $\mathrm{Ps}_{2}$ with $\mathrm{H}_{2}$ in its ground state. It could be however possible to form $(\mathrm{PsH})_{2}$, or to observe a signature resonance in a scattering experiment, when $\mathrm{Ps}_{2}$ collides with a highly vibrationally excited $\mathrm{H}_{2}$ if its wave function overlaps with some vibrational state of $(\mathrm{PsH})_{2}$, provided that the lifetime of this molecule, considering the electronpositron annihilation, is long enough. Yet another hypothesis could be the formation of this system using positron beams with the direct reaction $2 \mathrm{e}^{+}+2 \mathrm{H}^{-} \rightarrow(\mathrm{PsH})_{2}$ maybe using metal hydrides.

We hope that the present work will stimulate further theoretical research on this molecule and hopefully the development of new experiments to unveil the fascinating physics of this peculiar system.

If the hydrogen molecule is stripped of one electron it forms the simplest known molecule $\mathrm{H}_{2}^{+}$. Following the previous loose analogy between $\mathrm{PsH}$ and $\overline{\mathrm{H}}$, the analogous of the $\overline{\mathrm{H}}^{+}$ion (the bare antiproton) is $\mathrm{H}^{-}$, obtained removing one positron from $\mathrm{PsH}$. And indeed, the reaction $\overline{\mathrm{H}}+\overline{\mathrm{H}}^{+} \rightarrow \overline{\mathrm{H}}_{2}^{+}$has the analogous reaction $\mathrm{H}^{-}+\mathrm{PsH} \rightarrow \mathrm{e}^{+}\left(\mathrm{H}^{-}\right)_{2}$ which forms a stable species as recently shown by Charry et al. ${ }^{26}$ and confirmed by Ito et al. ${ }^{31}$ and Bressanini ${ }^{32}$.

The hydrogen atom can capture an electron to form the stable hydrogen negative ion $\mathrm{H}^{-}$. Similarly, $\mathrm{PsH}$ can capture a positron to form $\mathrm{e}^{+} \mathrm{PsH}{ }^{16}$.

Whether or not this analogy holds true for larger systems, like the analogous of the $\mathrm{H}_{3}^{+}$ion, remains to be seen, but we believe it is an intriguing question.

In his 1933 essay "The chemical bond" (oddly without a single reference), Gilbert N. Lewis ${ }^{33}$ wrote that "Not only is the pair of electrons not necessarily a bond, but the bond need not always be a pair". We may add that sometimes electrons are not even necessary, and in some cases their role can be played by positrons.

\section{ACKNOWLEDGMENTS}

I wish to thank Gabriele Morosi for many fruitful discussions.

\section{DATA AVAILABILITY}

The data supporting the findings of this study are available from the corresponding author upon request. 


\section{REFERENCES}

${ }^{1}$ R. Weissleder, and M. J. Pittet, Nature 452, 580 (2008).

2 J. Mayer, C. Hugenschmidt, and K. Schreckenbach, Phys. Rev. Lett. 105, 207401 (2010).

${ }^{3}$ M. Aguilar, L. A. Cavasonza, G. Ambrosi, L. Arruda, N. Attig, P. Azzarello, A. Bachlechner, F. Barao, A. Barrau, and L. Barrin, Phys. Rev. Lett. 122, 041102 (2019).

${ }^{4}$ C. M. Surko, G. Gribakin, and S. J. Buckman, J. Phys. B; At., Mol. Opt. Phys. 38, R57 (2005).

${ }^{5}$ G. N. Lewis, J. Am. Chem. Soc. 38, 762 (1916).

${ }^{6}$ W. Heitler, and F. London, Z. Phys. 44, 455 (1927).

${ }^{7}$ L. Zhao, W. H. E. Schwarz, and G. Frenking, Nat. Rev. Chem. 3, 35 (2019).

${ }^{8}$ C. D. Anderson, Phys. Rev. 43, 491 (1933).

${ }^{9}$ C. D. Anderson, Nature 133, 313 (1934).

${ }^{10}$ P. A. M. Dirac, Proc. R. Soc. London, Ser. A 133, 60 (1931).

${ }^{11}$ G. a. Baur, G. Boero, A. Brauksiepe, A. Buzzo, W. Eyrich, R. Geyer, D. Grzonka, J. Hauffe, K. Kilian, and M. LoVetere, Phys. Lett. B 368, 251 (1996).

${ }^{12}$ Alpha Collaboration, Nature 578, 375 (2020).

${ }^{13}$ A. Ore, Phys. Rev. 83, 665 (1951).

${ }^{14}$ D. M. Schrader, F. M. Jacobsen, N.-P. Frandsen, and U. Mikkelsen, Phys. Rev. Lett. 69, 57 (1992).

${ }^{15}$ D. Bressanini, and G. Morosi, J. Chem. Phys. 119, 7037 (2003).

${ }^{16}$ K. Varga, Phys. Rev. Lett. 83, 5471 (1999).

${ }^{17}$ D. Bressanini, Phys. Rev. A 99, 022510 (2019).

${ }^{18}$ S. L. Saito, Chem. Phys. Lett. 419, 589 (2006).

${ }^{19}$ J. Mitroy, and G. Ryzhikh, J. Phys. B; At., Mol. Opt. Phys. 32, L621 (1999).

${ }^{20}$ N. Jiang, and D. M. Schrader, Phys. Rev. Lett. 81, 5113 (1998).

${ }^{21}$ D. Bressanini, Phys. Rev. Lett. 109, 223401 (2012).

${ }^{22}$ D. Bressanini, Phys. Rev. A 97, 012508 (2018).

${ }^{23}$ D. Bressanini, and G. Morosi, J. Phys. B; At., Mol. Opt. Phys. 41, 145001 (2008).

${ }^{24}$ D. Bressanini, G. Morosi, and M. Mella, J. Chem. Phys. 116, 5345 (2002).

${ }^{25}$ S. C. Wang, Phys. Rev. 31, 579 (1928).

${ }^{26}$ J. Charry, M. T. d. N. Varella, and A. Reyes, Angew. Chem., Int. Ed. 57, 8859 (2018).

${ }^{27}$ M. Goli, and S. Shahbazian, ChemPhysChem 20, 831 (2019).

${ }^{28}$ K. Ruedenberg, Rev. Mod. Phys. 34, 326 (1962).

${ }^{29}$ D. B. Cassidy, and A. P. Mills Jr, Nature 449, 195 (2007).

${ }^{30}$ D. B. Cassidy, S. H. M. Deng, and A. P. Mills Jr, Phys. Rev. A 76, 062511 (2007).

${ }^{31}$ S. Ito, D. Yoshida, Y. Kita, and M. Tachikawa, J. Chem. Phys. 153, 224305 (2020).

${ }^{32}$ D. Bressanini, J. Chem. Phys. 154, 224306 (2021).

${ }^{33}$ G. N. Lewis, J. Chem. Phys. 1, 17 (1933). 


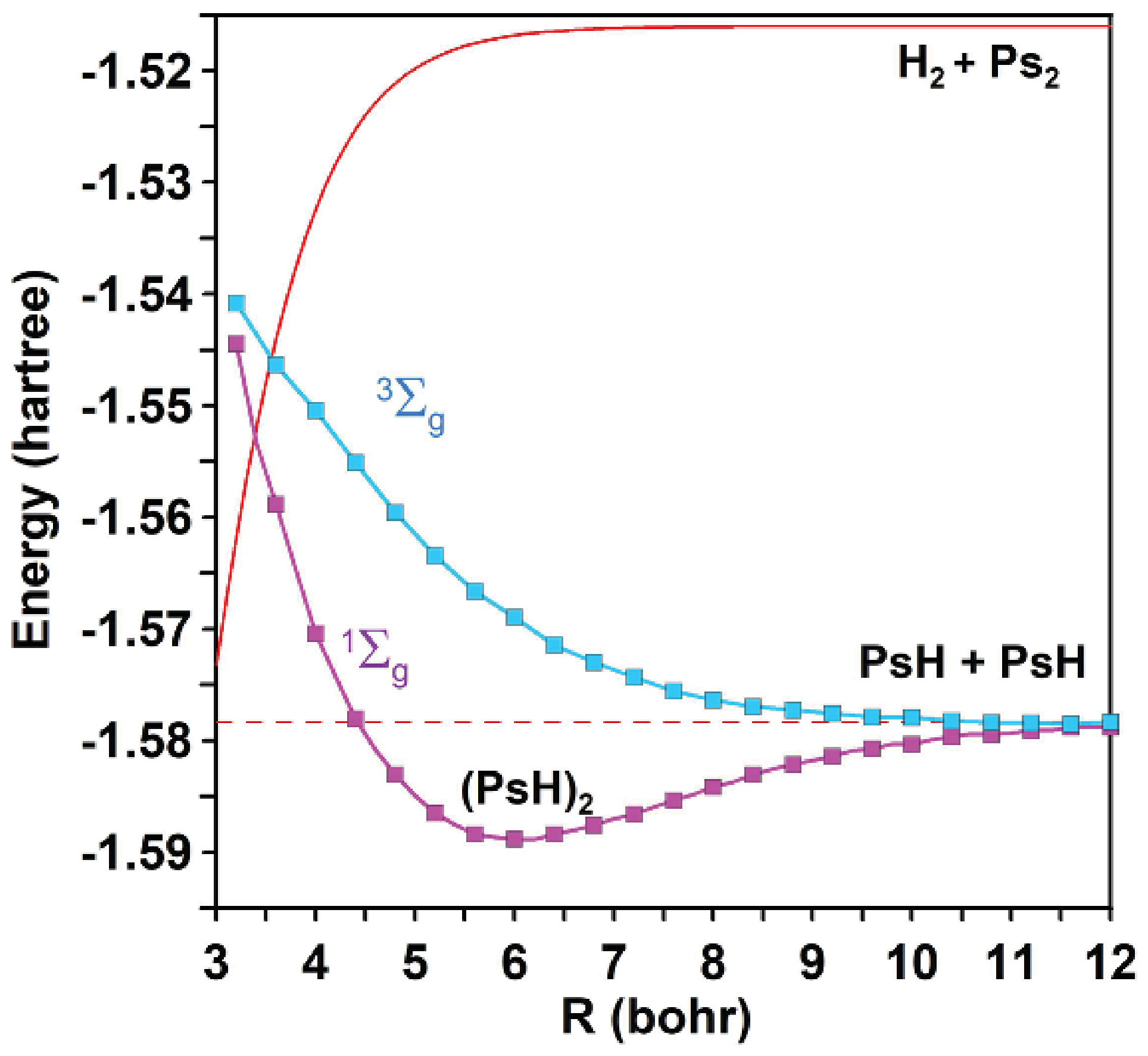




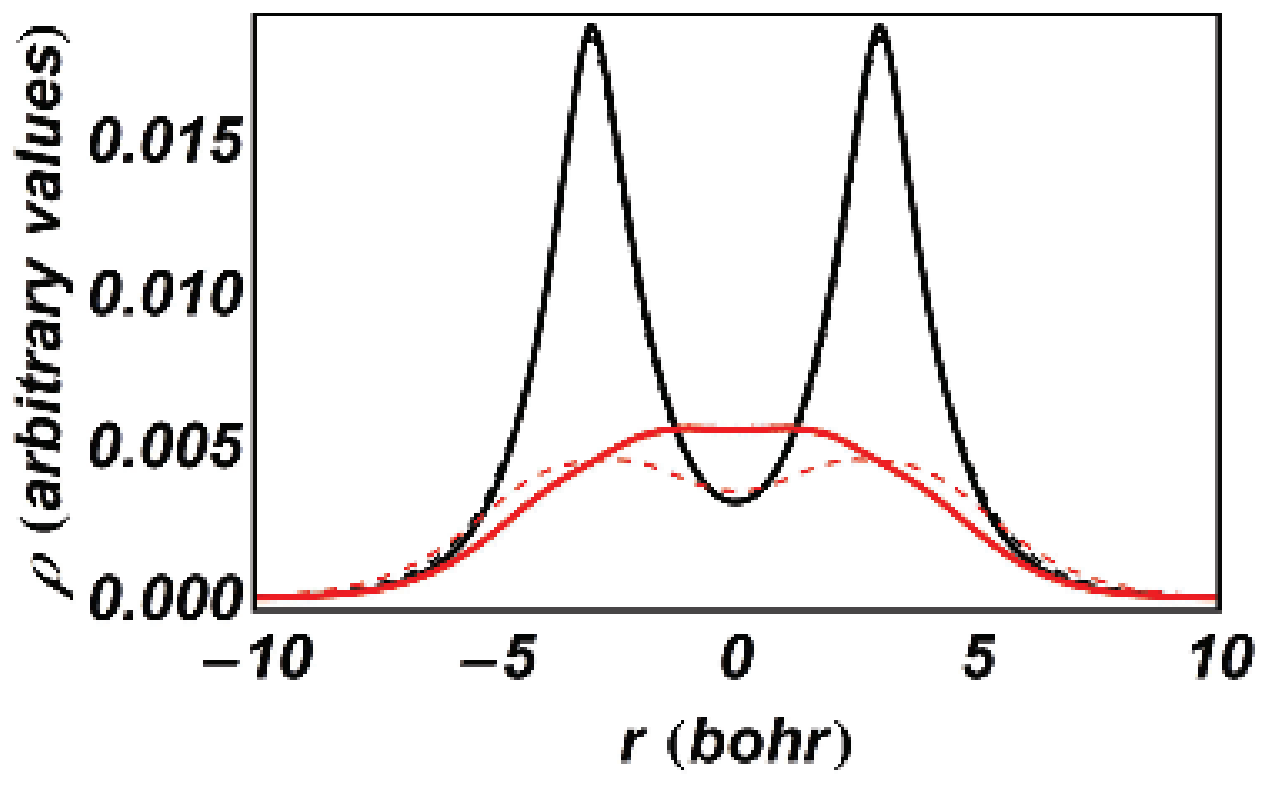



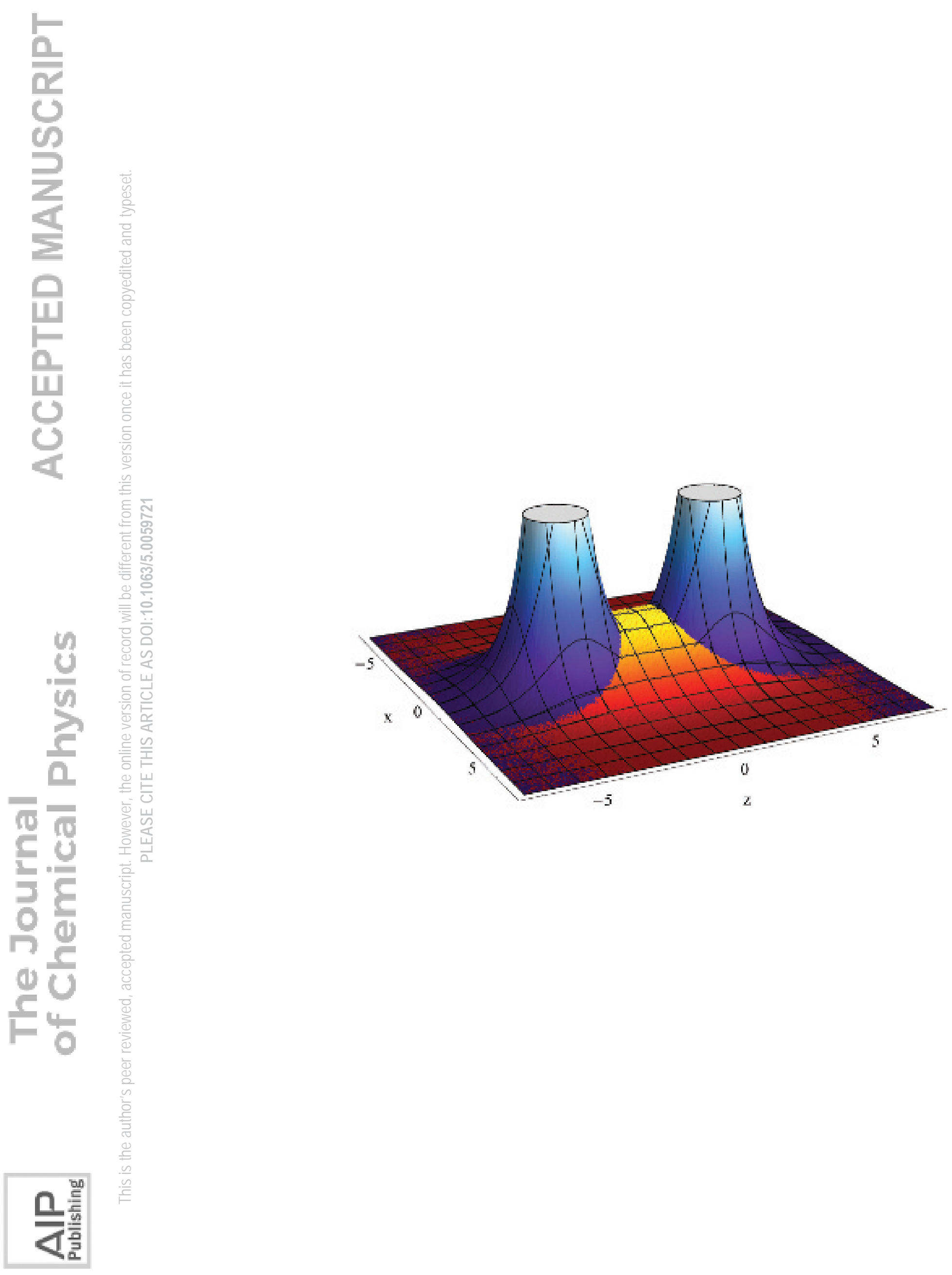